\title{
QUANTIZATION AND REGULARIZATION IN PERTURBATIVE QUANTUM COSMOLOGY
}

\author{
Giampiero Esposito \\ International Centre for Theoretical Physics, \\ Strada Costiera 11, 34014 Trieste, Italy \\ Scuola Internazionale Superiore di Studi Avanzati, \\ Via Beirut 2-4, 34013 Trieste, Italy
}

\begin{abstract}
Reduction to physical degrees of freedom before quantization leads to predictions for one-loop amplitudes in quantum cosmology in the presence of boundaries which disagree with the results obtained from Faddeev-Popov theory and boundary-counterterms technique. However, the mode-by-mode analysis of eigenvalue equations for gauge modes and ghost fields remains a very difficult problem. Hence the equivalence or inequivalence of various quantization and regularization techniques cannot be easily proved.

PACS 03.70. $+\mathrm{k}$ - Theory of quantized fields.

PACS 04.60.+n - Quantum theory of gravitation.
\end{abstract}

SISSA Ref. 4/93/A (January 1993) 
Quantization and Regularization in Perturbative Quantum Cosmology

In the last few years, much work has been done on perturbative properties of physical theories in the presence of boundaries within the framework of one-loop quantum cosmology [1]. The corresponding problems are as follows.

(i) Choice of locally supersymmetric boundary conditions [1]: they involve the normal to the boundary and the field for spin $\frac{1}{2}$, the normal to the boundary and the spin- $\frac{3}{2}$ potential for gravitinos, Dirichlet conditions for real scalar fields, magnetic or electric field for electromagnetism, mixed boundary conditions for the four-metric of the gravitational field (and in particular Dirichlet conditions on the perturbed three-metric).

(ii) Quantization techniques: one-loop amplitudes can be evaluated by first reducing the classical theory to the physical degrees of freedom (hereafter referred to as PDF) by choice of gauge and then quantizing, or by using the gauge-averaging method of Faddeev and Popov, or by applying the extended-phase-space Hamiltonian path integral of Batalin, Fradkin and Vilkovisky [1].

(iii) Regularization techniques: the generalized Riemann zeta-function and its regularized $\zeta(0)$ value (which yields both the scaling of the one-loop prefactor and the one-loop divergences of physical theories) can be obtained by studying the eigenvalue equations obeyed by perturbative modes, once the corresponding degeneracies and boundary conditions are known, or by using geometrical formulae for one-loop counterterms which generalize well-known results for scalar fields, but make no use of mode-by-mode eigenvalue conditions and degeneracies.

It turns out that one-loop quantum cosmology may add further evidence in favour of different approaches to quantizing gauge theories being inequivalent. Studying flat 
Quantization and Regularization in Perturbative Quantum Cosmology

Euclidean backgrounds bounded by a three-sphere, for electromagnetism the PDF method yields $\zeta(0)=-\frac{77}{180}$ and $\zeta(0)=\frac{13}{180}$ in the magnetic and electric cases respectively [1], whereas the indirect Faddeev-Popov method (i.e. when one-loop amplitudes are expressed using the boundary-counterterms technique and evaluating the various coefficients as in ref.[2]) is found to yield $\zeta(0)=-\frac{38}{45}$ in both cases [1,3]. For $N=1$ supergravity, the PDF method yields partial cancellations between spin 2 and spin $\frac{3}{2}$ [1], whereas the indirect Faddeev-Popov method yields a one-loop amplitude which is even more divergent than in the pure-gravity case [2]. Finally, for pure gravity, the PDF method yields $\zeta(0)=-\frac{278}{45}$ in the Dirichlet case, whereas the indirect Faddeev-Popov method yields $\zeta(0)=-\frac{803}{45}$ [1,2]. Moreover, within the PDF approach, it is possible to set to zero on $S^{3}$ the linearized magnetic curvature. This yields a well-defined one-loop calculation, and the corresponding $\zeta(0)$ value is $\frac{112}{45}[1]$. By contrast, using the Faddeev-Popov formula, magnetic boundary conditions for pure gravity are ruled out [2].

It is therefore necessary to get a better understanding of the manifestly gauge-invariant formulae for one-loop amplitudes used so far in the literature, by performing a mode-bymode analysis of the eigenvalue equations, rather than relying on general formulae which contain no explicit information about degeneracies and eigenvalue conditions. As shown in ref.[1], this detailed analysis can be attempted for vacuum Maxwell theory studied at one-loop about flat Euclidean backgrounds bounded by a three-sphere, recently considered in perturbative quantum cosmology. Working within the Faddeev-Popov formalism and making a $3+1$ split of the vector potential, the full $\zeta(0)$ value takes into account the contribution of the physical degrees of freedom, i.e. the transverse part $A^{(T)}$ of the vector 
Quantization and Regularization in Perturbative Quantum Cosmology

potential, the gauge modes, i.e. the $A_{0}$ component and the longitudinal part $A^{(L)}$ of the vector potential, and the ghost action. Interestingly, a gauge-averaging term can be found such that the contributions to $\zeta(0)$ of physical degrees of freedom and of decoupled mode

for $A_{0}$ add up to $-\frac{61}{90}$ both in the electric and in the magnetic case. However, remaining modes for $A_{0}$ and $A^{(L)}$ are always found to obey a coupled system of second-order ordinary differential equations. This system has been solved exactly [1], but unfortunately the power series appearing in its solution are not (obviously) related to well-known special functions. The corresponding asymptotic analysis (i.e. at large values of the eigenvalues) is therefore much harder, and remains a stimulating challenge for applied mathematicians and theoretical physicists. It also turns out that, in the presence of boundaries, gauge modes should remain coupled, for us to be able to find a linear, elliptic second-order operator corresponding to the ghost action. Since the difficulties concerning gauge modes and ghost fields are technical in nature and not completely unfamiliar (i.e. systems of ordinary differential equations, fourth-order algebraic equations, finite parts of diverging series), there is hope that the research initiated in ref.[1] (see corrections in the Appendix) will shed new light on one-loop properties of physical theories in the presence of boundaries.

\section{APPENDIX}

Since we rely on ref.[1], we should correct the mistakes appearing therein. They are as follows. The comments (1), (2) and (3) following eq. (2.3.22) should read: There are secondary constraints, i.e. $q_{2} \approx 0$, and all constraints are second-class. A similar correction 
Quantization and Regularization in Perturbative Quantum Cosmology

should be made on page 37 (see ref.[4]). On page 64 , the exponential $e^{\frac{r}{2 M}}$ on the righthand side of eqs. (3.3.14)-(3.3.15) should read $e^{\frac{r}{4 M}}$. On page 170, the second line following eq. (6.5.43) should read: ..., the partial effect of the ghost field, and the effect of the $R_{1^{-}}$ mode. Indeed, there are additional contributions since the gauge-averaging functional of eq. (6.5.9) is not the Lorentz functional ${ }^{(4)} \nabla^{\mu} A_{\mu}$.

\section{REFERENCES}

[1] G. ESPOSITO: Quantum Gravity, Quantum Cosmology and Lorentzian Geometries, Lecture Notes in Physics, New Series m: Monographs, Volume m12 (Springer-Verlag, Berlin, 1992).

[2] S. POLETTI: Phys. Lett., B 249, 249 (1990).

[3] I. G. MOSS and S. POLETTI: Phys. Lett., B 245, 355 (1990).

[4] A. CORICHI: J. Math. Phys., 33, 4066 (1992). 